Parkhomenko K. Yu. Laparoscopic simultaneous operations in patients with hernias of various location and comorbid pathology. Journal of Education, Health and Sport. 2020;10(4):351-356. eISSN 2391-8306. DOI http://dx.doi.org/10.12775/JEHS.2020.10.04.038 https://apcz.umk.pl/czasopisma/index.php/JEHS/article/view/JEHS.2020.10.04.038

https://zenodo.org/deposit/4553592



Received: 06.04.2020. Revised: 23.04.2020. Accepted: 30.04.2020.

\title{
LAPAROSCOPIC SIMULTANEOUS OPERATIONS IN PATIENTS WITH HERNIAS OF VARIOUS LOCATION AND COMORBID PATHOLOGY
}

\author{
K. Yu. Parkhomenko
}

\section{Kharkiv National Medical University}

\begin{abstract}
Aim. The study of the frequency of concomitant surgical pathology and simultaneous operations during videolaparoscopic treatment of hernias of different localization and evaluate of the results of surgical treatment of patients with comorbid pathology.

Materials and methods. A prospective study was conducted on 222 patients with comorbid pathology. All patients were divided into two groups. The main group included 120 patients with hernias, who in addition to videolaparoscopic hernioplasty, performed simultaneous operations. The comparison group included 102 patients who performed only videolaparoscopic hernioplasty. In the course of this study, the following parameters were evaluated: frequency and structure of simultaneous operations, duration of operations, intraoperative and early postoperative complications.

Results. Comorbid pathology was diagnosed in 146 patients (52.3\%). These patients underwent 194 simultaneous operations: cholecystectomy - 91 (46.9\%); adnexectomy - 14 $(7.2 \%)$; hysterectomy $-12(6.1 \%)$; varicocelectomy $-8(4.1 \%)$; adhesiolysis $-49(25.2 \%)$; appendectomies - $13(6.7 \%)$; fenestration of cysts of the liver or mesentery $-7(3.8 \%)$.

Conclusion. The effectiveness of simultaneous operations is not limited to purely economic indicators, it is projected to affect during the postoperative period.
\end{abstract}


Keywords: simultaneous operations; comorbid pathology; laparoscopic surgery; hernioplasty.

Introduction. In recent years, the number of patients with comorbid pathology has increased. The incidence of comorbidity is almost three times higher in patients older than 55 years than in the general population $[1,3,5]$. In such patients, combined pathology of the digestive tract is more often diagnosed. However, according to the literature, the number of patients with concomitant gynecological and urological pathologies has recently increased. The total number of patients with comorbid pathology is 25-30\% [2]. In such cases, simultaneous surgical treatment of several diseases is the best treatment option $[2,4]$.

This approach can increase the cosmetic effect after surgery and reduce the risk of postoperative complications and economic costs of treatment.

Improvement of diagnostic methods and development of endoscopic methods of surgical treatment have increased the possibility of performing simultaneous operations. However, analysis of the literature indicates that the frequency of such operations does not exceed $6 \%[1,3,4]$. The interest of modern surgeons in laparoscopic simultaneous operations is explained by their advantages. The relevance of this topic lies in the absence of clear indication for operations, their classification and insufficient data on long-term treatment results [2].

Objective. The study of the frequency of concomitant surgical pathology and simultaneous operations during videolaparoscopic treatment of hernias of different localization and purpose of this study was to evaluate of the results of surgical treatment of patients with comorbid pathology.

Materials and methods. A prospective study was conducted on 279 patients with hernias of various location and comorbid pathology. The age of the patients varied from 25 to 72 years. The study group included patients with hernias of various localization, chronic appendicitis, cholelithiasis, and other gynecological and urological pathologies. All patients were treated in the surgical departments of the Kharkiv regional hospital and Specialized medical institution №13 from December 2016 to January 2018. All patients were divided into two groups. The main group included 146 patients with hernias, who in addition to videolaparoscopic hernioplasty, performed simultaneous operations. The comparison group included 133 patients who performed only videolaparoscopic hernioplasty. In the course of this study, the following parameters were evaluated: frequency and structure of simultaneous operations, duration of operations, intraoperative and early postoperative complications. After 
the diagnosis was made, the patients were subjected to laboratory and instrumental examinations. Laboratory methods were presented with a standard set of analyzes. Instrumental examination is represented by a comprehensive ultrasound of the abdominal organs cavity, extraperitoneal space and cardio-vascular system, electrocardiography and research functions of external respiration; also 67 (24\%) patients underwent computed tomography.

The operations were performed using general anesthesia and artificial lung ventilation (33.6\%) and spinal anesthesia (66.4\%). Operations on axillary hernias were performed by TAPP (Trans Abdominal Pre-Peritoneal); umbilical and postoperative hernia - IPOM method (intraperitoneal onlay mesh). For plastic hernia gate was used mesh polypropylene or composite implant. We also performed laparoscopis fundoplication to correct hernia of the esophageal orifice of the diaphragm. In all cases, operational access was provided in typical places. The results were analyzed using statistical programs PSSR; for the analysis of qualitative data used the criterion $\chi^{2}$; for the evaluation quantitative data $-\mathrm{t}$-criterion and criterion Kolmogorov-Smirnov.

Results. The structure of hernias was presented as follows: in 84 patients $(30.1 \%$ of cases) inguinal hernias, in 52 patients $(24.3 \%)$ - postoperative, in 45 patients $(18.2 \%)$ umbilical, in 34 patients $(12.1 \%)$ - femoral hernias, in 42 patients $(15.3 \%)$ - hernia of the esophageal orifice of the diaphragm.

Comorbid pathology was diagnosed in 146 patients $(52.3 \%)$. These patients underwent 194 simultaneous operations: cholecystectomy - 91 (46.9\%); adnexectomy - 14 (7.2\%); hysterectomy - $12(6.1 \%)$; varicocelectomy - 8 (4.1\%); adhesiolysis - 49 (25.2\%); appendectomies $-13(6.7 \%)$; fenestration of cysts of the liver or mesentery $-7(3.8 \%)$.

Concomitant diseases were detected at the stage of preoperative examination in all cases. Therefore, all simultaneous surgeries were planned interventions and had predicted minimal risks to patient's health. Thus, the total number of patients was 279 person, the total number of operations was 398. For each of the 146 patients, simultaneous operations performed an average of 2.4 laparoscopic operations, including 2 operations performed on 92 patients, $3-27$ patients.

The duration of operations of the main group was greater on average by $29.4 \pm 13$ min (from $13 \mathrm{~min}$ in fenestration of cysts to $94 \mathrm{~min}$ in laparoscopic hysterectomy) and was (95.7 \pm 19.2 ) compared with $66.7 \pm 14.6 \mathrm{~min}$ in patients of the comparison group ( $p<0,05)$.

Intraoperative and early postoperative complications occurred in $6(2.7 \%)$ patients. Thus, in 1 case - intraperitoneal bleeding after performing isolated hernioplasty; in 1 cases - 
thromboembolism of small branches of the pulmonary artery when performing simultaneous hernioplasty with laparoscopic fundoplication; in 2 cases - preperitoneal hematoma, in 2 seroma in the area trocar access. The frequency of complications of the group did not differ significantly: $2.9 \%$ - in isolated hernioplasty and $2.5 \%$ for simultaneous operations $\left(\chi^{2}=0.045\right.$; $\mathrm{p}<0,05)$.

The most frequent simultaneous operations during laparoscopic hernioplasty were cholecystectomy and appendectomies. The reason for performing simultaneous operations was gynecological and urological pathology, which accounted for $19.8 \%$ of the total number of operations.

Simultaneous operations are not accompanied by an increase in the frequency of intraoperative and early postoperative complications. Although the duration of surgery was longer, it did not lead to an increase in the length of stay of patients in the surgical department, which was on average $4.2 \pm 0.8$ bed-day compared with $3.4 \pm 0.7$ after isolated videolaparoscopic hernioplasty $(\mathrm{p}<0,05)$.

Results. In all patients with hernias of different localization is necessary thorough preoperative examination. This will ensure the detection of pathology of the abdominal cavity and retroperitoneal space, even in the absence of clinical manifestations. Simultaneous operations minimize the trauma of surgery, recovery and rehabilitation of patients. The effectiveness of such operations is not limited to purely economic indicators, it is projected to affect during the postoperative period. Use of an individual approach to laparoscopic treatment of patients with hernias of different localization and comorbid pathology is guaranteed to reduce the degree of surgical risk.

\section{References:}

1. Akinci M., Yilmaz K., Kulah B. Association of ventral incisional hernias with comorbid diseases. Chirurgia. 2013. - 108(6). - P. 807-811.

2. Arafat S., Alsabek M. Simultaneous laparoscopic cholecystectomy and transabdominal preperitoneal hernioplasty: two cases reports evaluate the safety and surgical complicated. Clin Case Rep. - 2017. - 5(12). - P. 2093-2096.

3. Lehmann A., Piatcowski J., Nowak M. Simultaneous TAPP (transabdominal preperitoneal technique) for inguinal hernia and cholecystectomy - a feasible and safe procedure. Pol Przegl Chir. - 2014. - 86(2). - P. 73-76.

4. Orr N.T., Davenport D.L., Roth S.S. Outcomes of simultaneous laparoscopic cholecystectomy and ventral hernia repair compared to that of laparoscopic cholecystectomy alone. Surg Endosc. - 2013. - 27(1). - P. 67-73. 
5. Kamer E. Laparoscopic cholecystectomy accompanied by simultaneous umbilical hernia repair: a retrospective study. JPGM. - 2013. - 53(3). - P. 176-180.

6. Plymale M.A., Ragulojan R., Davenport D.L., Roth J.S. Ventral and incisional hernia: the cost of comorbidities and complications. Surg Endosc. 2017 Jan;31(1):341-351. doi: 10.1007/s00464-016-4977-8.

7. Holihan J.L., Alawadi Z., Martindale R.G., et al. Adverse events after ventral hernia repair: the vicious cycle of complications. J Am Coll Surg. 2015. - Aug; 221(2):47885. doi: 10.1016/j.jamcollsurg.2015.04.026.

8. Le Gall H., Reibel N., De Runz A., et al. Abdominoplasty and simultaneous laparoscopic ventral hernia repair. Clinical study about 45 patients. Ann Chir Plast Esthet. 2017 Apr; 62(2):115-121. doi:10.1016/j.anplas.2016.06.005.

9. Filho J.M., Maciel S., Belerique M. Abdominoplasty combined with intraabdominal gynecologic surgery. Rev. Soe. Bras. CiroPlást. 2004; 19(2): 41-52.

10. Cheesborough J.E., Dumanian G.A. Simultaneous prosthetic mesh abdominal wall reconstruction with abdominoplasty for ventral hernia and severe rectus diastasis repairs. Plast. Reconstr. Surg. 2015; 135: 268-276. DOI: 10.1097/PRS.0000000000000840.

11. Koolen P.G., Ibrahim A.M., Kim K., et al. Patient selection optimization following combined abdominal procedures: analysis of 4925 patients undergoing panniculectomy/abdominoplasty with or without concurrent hernia repair. Plast Reconstr Surg. 2014 Oct; 134(4):539e-50e. doi: 10.1097/PRS.0000000000000519.

12. Ortega J., Navarro V., Cassinello N., Lledo S. Requirement and postoperative outcomes of abdominal panniculectomy alone or in combination with other procedures in a bariatric surgery unit. Am J Surg. 2010; 200:235-240. https ://doi.org/10.1016/j.amjsurg.2009.07.043.

13. Warren J.A., Epps M., Debrux C., et al. Surgical site occurrences of simultaneous panniculectomy and incisional hernia repair. Am Surg. 2015 Aug; 81(8):764-9.

14. Zemlyak A.Y., Colavita P.D., El Djouzi S., et al. Comparative study of wound complications: isolated panniculectomy versus panniculectomy combined with ventral hernia repair. J Surg Res. 2012 Oct;177(2):387-91. doi: 10.1016/j.jss.2012.06.029.

15. Wadhwa A., Chowbey P.K., Sharma A., et al. Combined procedures in laparoscopic surgery. 2003 Dec;13(6):382-6. doi: 10.1097/00129689-200312000-00007.

16. Kim G., Lomanto D., Lawenko M.M., et al. Single-port endo-laparoscopic surgery in combined abdominal procedures. Asian J End Surg. 2013; 6(3): 209-213.

17. Hayakawa S., Hayakawa T., Inukai K., et al. Simultaneous transabdominal 
preperitoneal hernia repair and laparoscopic cholecystectomy: A report of 17 cases. Asian $\mathbf{J}$ Endosc Surg. 2018 Nov 8. doi: 10.1111/ases.12667.

18. Quezada N., Maturana G., Pimentel E., et al. Simultaneous TAPP inguinal repair and laparoscopic cholecystectomy: results of a case series. Hernia. 2019 Feb;23(1):119-123. doi: 10.1007/s10029-018-1824-y.

19. Schulster M.L., Cohn M.R., Najari B.B., Goldstein M. Microsurgically assisted inguinal hernia repair and simultaneous male fertility procedures: rationale, technique and outcomes. J Urol. 2017 Nov;198(5):1168-1174. doi: 10.1016/j.juro.2017.06.072.

20. Benlice C., Gorgun E., Aytac E., et al. Mesh herniorrhaphy with simultaneous colorectal surgery: a case-matched study from the American College of Surgeons National Surgical Quality Improvement Program. Am J Surg. 2015 Oct; 210(4):766-71. doi: 10.1016/j.amjsurg.2015.04.018. 